\title{
Mechanical Properties and Machinability of Glass Fiber-Reinforced Polyetheretherketone
}

\author{
S. J. Ji, H. J. Yu, J. Zhao, ${ }^{1}$ and F. S. Liang \\ School of Mechanical Science and Engineering, Jilin University, Changchun, China \\ 1 jzhao@jlu.edu.cn
}

Polyetheretherketone (PEEK) is a new type of special engineering plastic that has broad market potentials due to its particular microstructure and mechanical properties. However, few data can be found in the literatures devoted to the mechanical properties and machinability of PEEK, especially in the superprecision field. For the first time, the microscopic mechanical properties and machinability of glassfiber-reinforced PEEK (GF/PEEK), which is one typical derivative of pure PEEK, are studied. The nanoindentation experiment was performed to analyze the microscopic mechanical properties of GF/PEEK. The machinability was studied by single-point diamond turning (SPDT) on the GF/PEEK surface, the roughness and form accuracy of the machined surface were obtained in the following test. Experimental results indicate that GF/PEEK has good microscopic mechanical properties and machinability.

Keywords: glass-fiber-reinforced polyetheretherketone, micromechanical properties, nanoindentation, machinability, single-point diamond turning.

Introduction. Polyetheretherketone is a relatively new ultra-high performance thermoplastic with excellent high-temperature properties and exceptional characteristics of wear resistance and high specific strength. Hence, PEEKs have been widely recognized as the prospective materials for an extensive range of applications, such as the aerospace industry, automobile manufacturing and medical field [1-4].

To meet the requirements of high-precision, heat-resisting and high-fatigue strength in manufacturing, PEEK composite materials with further enhanced properties can be obtained by reinforcing with glass or carbon fibers [5-7]. The glass-fiber-reinforced PEEK (GF/PEEK), which is a typical derivative of PEEK, can also enhance the tensile and bending strengths of materials [5].

There is a wide variety of studies on fiber-reinforced PEEK, but these are mainly concentrated on several aspects, such as crystallization behavior [8, 9], morphology analysis [10], and macroscopic mechanical properties [11-13]. However, only few papers are focused on the micromechanical properties and machinability of fiber-reinforced PEEK.

PEEK is commonly manufactured by traditional methods of molding and processing for thermoplastic plastics, such as extrusion molding, injection molding and compression molding. The machined workpieces need additional post-processing to reach the super surface roughness and form accuracy [5, 14]. Among the available ultra-precision machining methods, the single-point diamond turning (SPDT) has been widely considered to be a promising technique due to the superior advantage of generating higher surface quality with only one processing step [15-17].

In this paper, the micromechanical properties and machinability for GF/PEEK are studied to obtain a better understanding of its characteristics. One of the major concerns in studying is to obtain the varying pattern for mechanical property and machining technology, thus making the excellent properties of PEEKs maximally applied.

1. Experiment and Results on Determination of Micromechanical Properties. Several GF/PEEK specimens were prepared in cylinder shapes with $R=8 \mathrm{~mm}$, and the surfaces were polished to make preparation for subsequent experiments. The morphology of the obtained GF/PEEK specimen is shown in Fig. 1. 


\begin{tabular}{|c|c|}
\hline $\begin{array}{l}\text { T a b } 1 \text { e } 1 \\
\text { Parameters Adopte } \\
\text { in the Nanoindentation Ex }\end{array}$ & \\
\hline Parameter & Value \\
\hline Surface approach velocity $(\mathrm{nm} / \mathrm{s})$ & 10 \\
\hline Peak hold time (s) & 2 \\
\hline Poisson's ratio & 0.3 \\
\hline
\end{tabular}

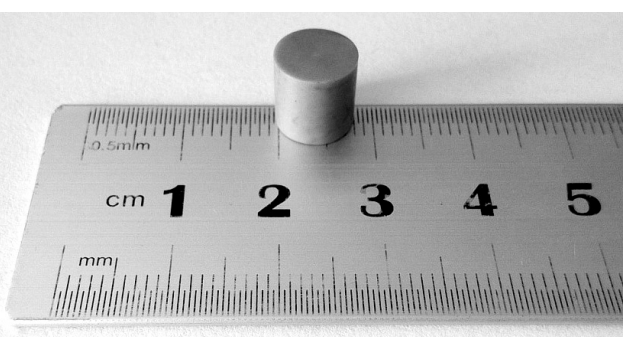

Fig. 1. The morphology of GF/PEEK.

The micromechanical properties are studied by a series of nanoindentation experiments, and the indenter of the adopted instrument is a triangular pyramid tip with $120^{\circ}$ apex angle. The parameters are presented in Table 1.

The experiments are performed at room temperature, and the purpose is to investigate the relation between indentation depth $h$ and load $P$. Five groups of nanoindentation experiments with different maximum indentation depth, including $1,2,4,6$, and $8 \mu \mathrm{m}$, are designed, and four test points in different locations are planned in each group. The microscope image of GF/PEEK after nanoindentation experiments with $8 \mu \mathrm{m}$ maximum indentation depth is given in Fig. 2. Taking the average values of four test points as the final dates, and the relation between measured indentation depth and load for different maximum depths is indicated in Fig. 3.

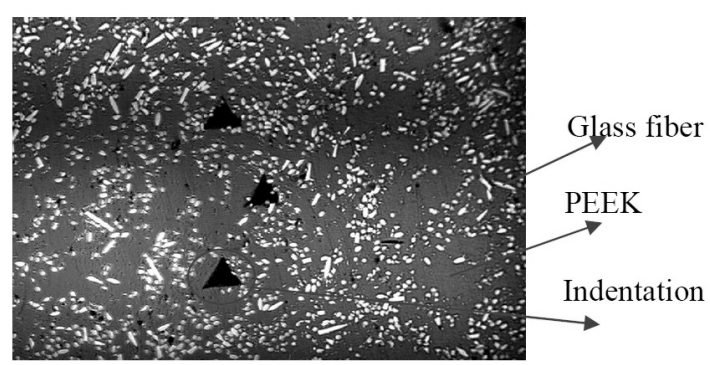

Fig. 2. The microscope image of GF/PEEK after nanoindentation ( $8 \mu \mathrm{m}$ maximum depth).

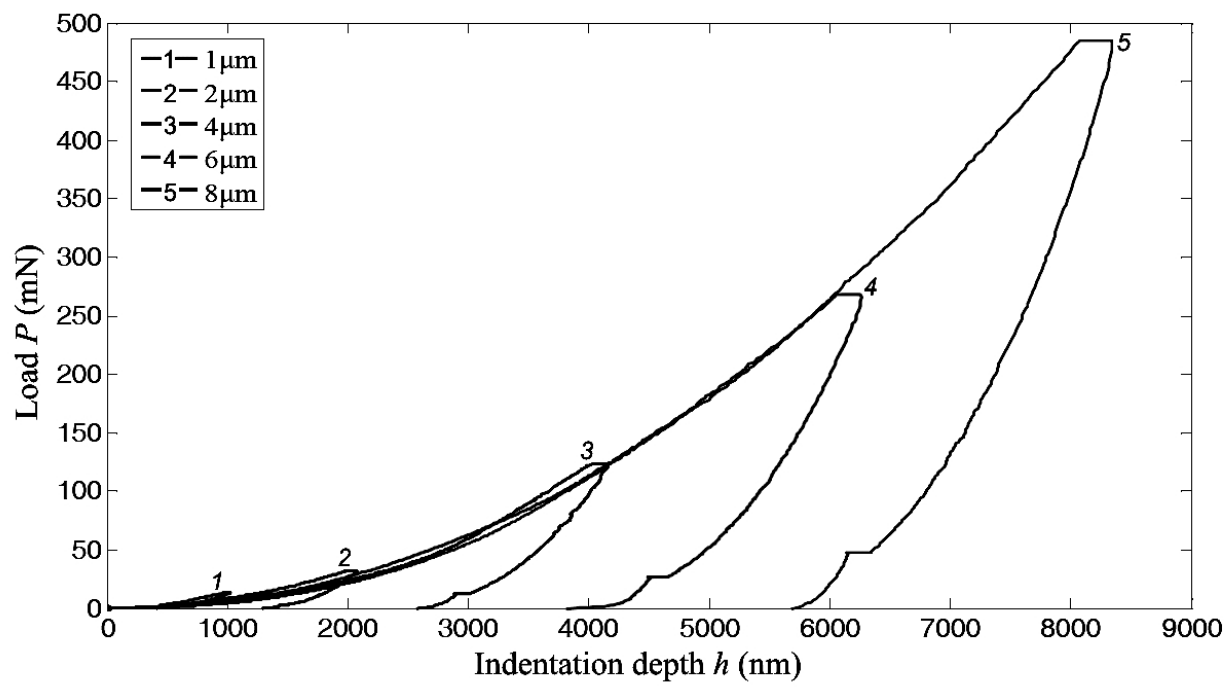

Fig. 3. Indentation depth vs. load for GF/PEEK. 
As shown in Fig. 2, the highlighted spots are glass fibers, and the triangular patches are indentations. At the conditions of $8 \mu \mathrm{m}$ maximum indentation depth, the GF/PEEK surface still keeps an excellent integrity, and it can also be seen that the indented surface is free of unwanted defects. From Fig. 3, it can be found that the load-depth curves have a similar trend for different groups, and the existing small difference may attribute to the inhomogeneous mixing for glass fibers among PEEK matrices. The results of nanoindentation experiments demonstrate that the GF/PEEK has superior micromechanical properties.

2. Experiment and Results on Machinability. The study of machinability for GF/PEEK is very essential and urgent for the wide application in some high-precision fields. To obtain a surface with high quality, SPDT technique is used to research the machinability of this thermoplastic plastic GF/PEEK. In addition, the feed rate adopted in SPDT is the microscale, and the study in Section 2 can demonstrate that the press in microscale cannot destroy this surface.

The machining experiment is carried out on a two-axis ( $X$ - and $Z$-axis) ultra-precision lath NANOFORM 250. Figure 4 displays a photograph of the main section of the experimental setup. During the cutting process, the GF/PEEK workpiece is fixed on the spindle through the air chuck and rotated with the spindle at a certain speed. The different cutting depth and speed can be achieved through the movement of $X$ - and $Z$-axis. The parameters of chosen diamond tool are listed in Table 2, and the geometry of employed diamond tool is presented in Fig. 5.

$\mathrm{T}$ a b 1 e 2

Parameters of Chosen Diamond Tool

\begin{tabular}{|c|c|c|c|c|}
\hline Parameter & $\begin{array}{c}\text { Radius } \\
(\mathrm{mm})\end{array}$ & $\begin{array}{c}\text { Rake angle } \\
(\mathrm{deg})\end{array}$ & $\begin{array}{c}\text { Clearance angle } \\
(\mathrm{deg})\end{array}$ & $\begin{array}{c}\text { Included angle } \\
(\mathrm{deg})\end{array}$ \\
\hline Value & 0.5 & 0 & 10 & 120 \\
\hline
\end{tabular}

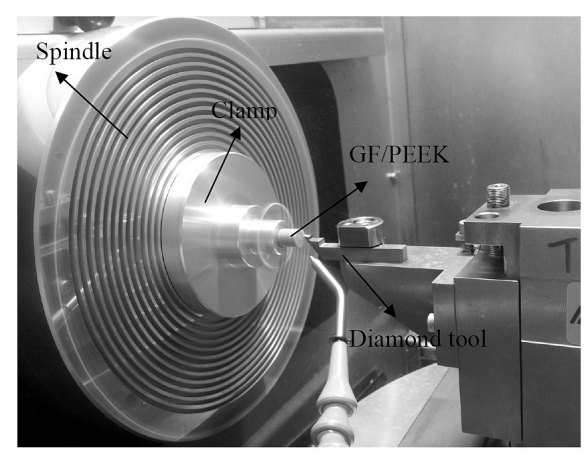

Fig. 4. Main section of the experiment setup.

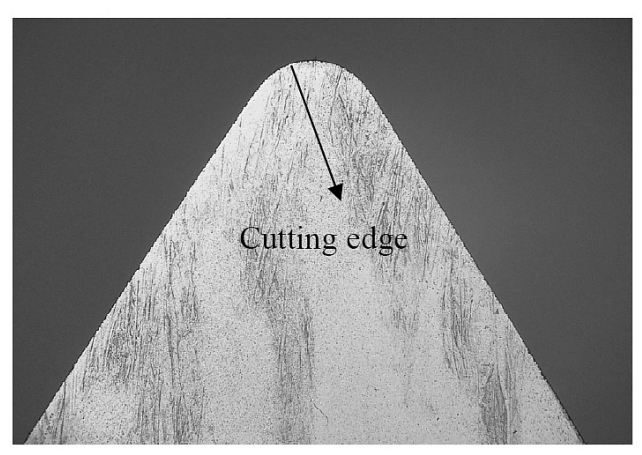

Fig. 5. The diamond tool geometry.

A simple flat surface is manufactured, and the spindle speed and feed rate are chosen to be $S=1500 \mathrm{rpm}$ and $F=1 \mathrm{~mm} / \mathrm{min}$, respectively. The surfaces are observed and measured by the TAYLOR HOBSON, which is a high-precision commercial instrument for the measuring of surface form, and mainly used to measure the form accuracy and surface roughness of the surface. The measurement is the typical contact measurement, and the operation is carried out by scanning a diamond stylus with a radius of $2 \mu \mathrm{m}$ in the radius direction of the machined surface. Figure 6 shows the outer shape of machined GF/PEEK surface and the schematic diagram of the measurement process. 


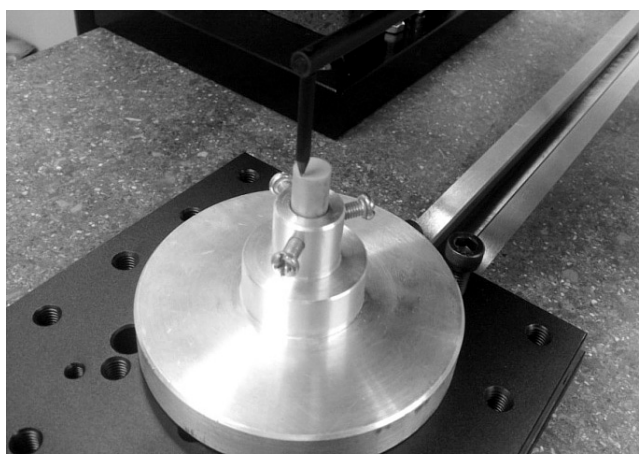

Fig. 6. Machined GF/PEEK surface and schematic diagram of the measurement process.

To compare the cutting effect of before and after the process, the blank surface and machined surface are measured by TAYLOR HOBSON, and the obtained raw profiles are firstly given in Fig. 7a and b, respectively. As indicated in Fig. 7, the machined surface has greatly enhanced the surface smoothness, as compared with the blank surface.

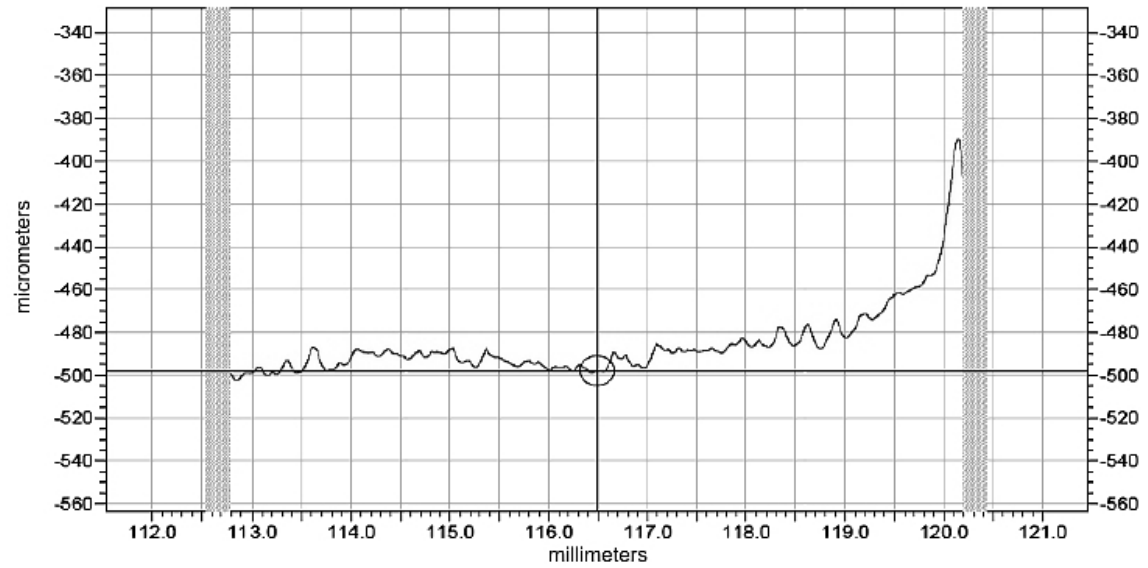

a

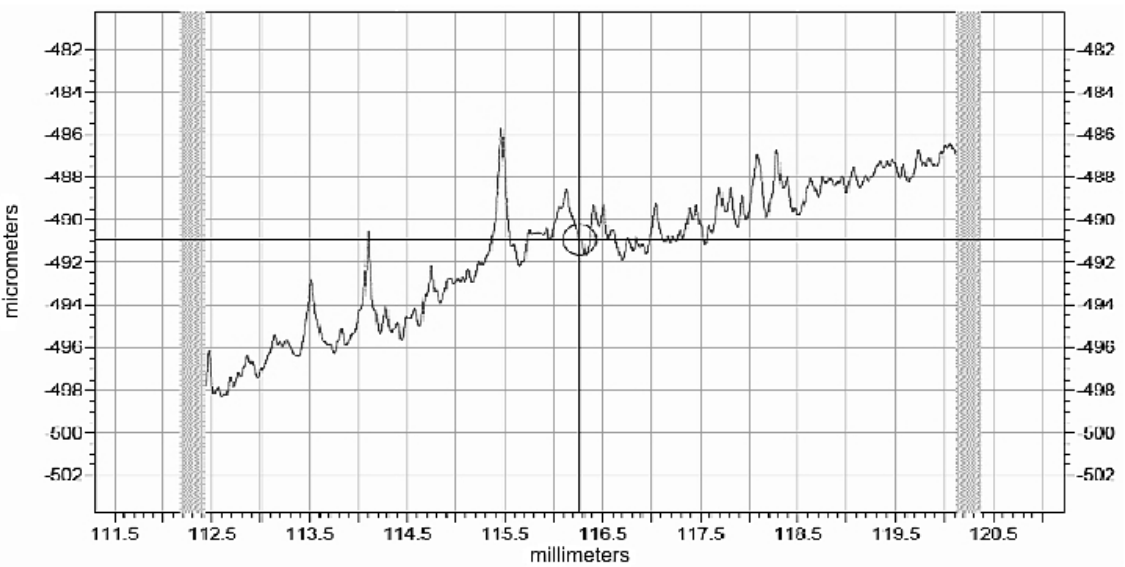

$\mathrm{b}$

Fig. 7. The raw profiles of GF/PEEK surface: (a) blank surface; (b) machined surface. 

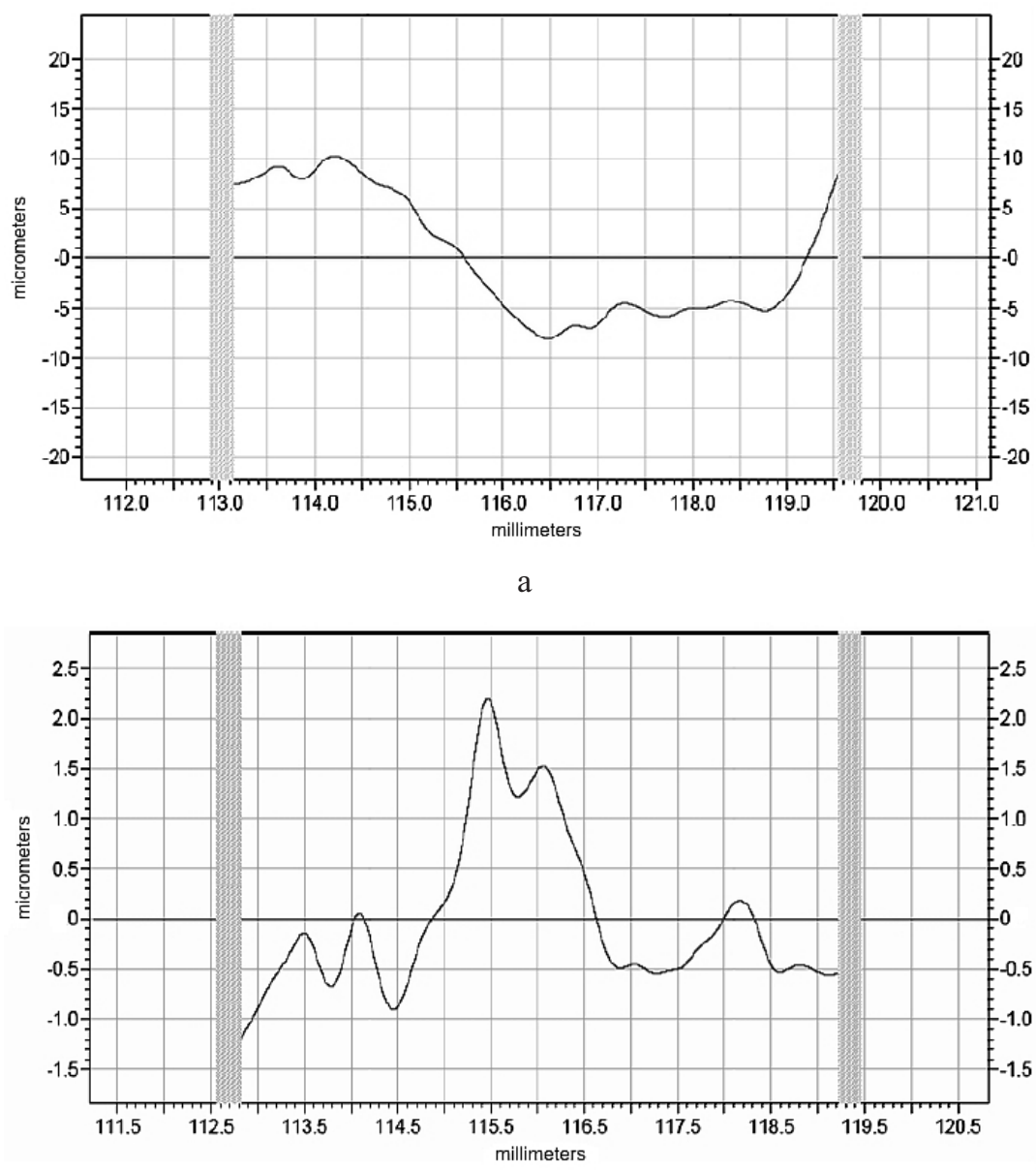

$\mathrm{b}$

Fig. 8. The form accuracy of GF/PEEK surface: (a) blank surface; (b) machined surface.

Based on the analysis module of the instrument for the raw profile, the analyses of form accuracy for blank surface and machined surface are displayed in Fig. 8. As indicated in Fig. 8a, the measuring form accuracy of blank surface is $18.3 \mu \mathrm{m}$ in peak to valley $(P V)$ value, while $P V$ for machined GF/PEEK surface drops to $3.4 \mu \mathrm{m}$ according to Fig. $8 \mathrm{~b}$. Furthermore, the analyses of surface roughness for blank surface and machined surface are shown in Fig. 9. The surface roughness $(R a)$ of blank is $1.77 \mu \mathrm{m}$, while the surface roughness of the machined surface is only $0.53 \mu \mathrm{m}$, as shown in Fig. 9b, which is not the superior level for the surface roughness in ultra-precision machining. The results may be attributed to the inhomogeneous mixing for glass fibers among PEEK matrices since the hard and brittle glass fibers may preclude the cutting and increase the cutting difficulty. The measurement results demonstrate that the ultra-precision machining strategy SPDT is suitable for the machining of thermoplastic plastic GF/PEEKs to generate a high-quality surface, and the GF/PEEK has an excellent machinability.

Conclusions. In this paper, the micromechanical properties and machinability of glass-fiber-reinforced polyetheretherketone (GF/PEEK) are analyzed to expand the application area. A series of nanoindentation experiments are conducted on the GF/PEEK surface to investigate the micromechanical properties. The results illustrate that the load-depth curves have a similar trend under different indentation depth conditions, which 


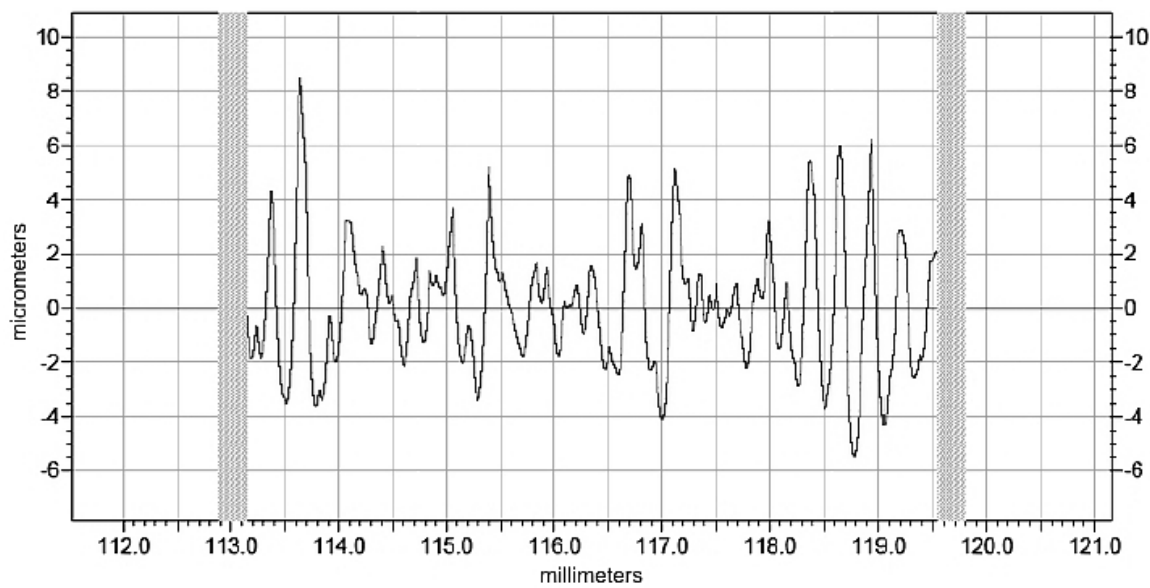

a

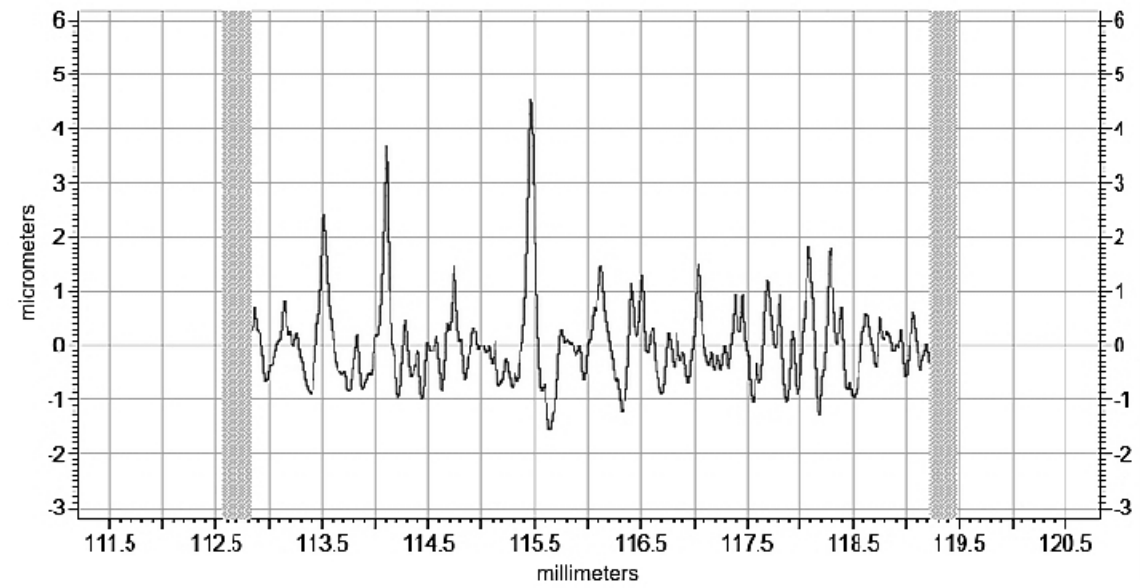

b

Fig. 9. Surface roughness of GF/PEEK surface: (a) blank surface; (b) machined surface.

demonstrates that the GF/PEEKs have excellent micromechanical properties. A flat surface is fabricated with SPDT to analyze the machinability of GF/PEEK in ultra-precision machining. From the measurement results, a microstructured surface with the form accuracy $P V=3.4 \mu \mathrm{m}$ and surface roughness $R a=0.53 \mu \mathrm{m}$ is obtained, which indicates that the GF/PEEKs also have an exellent machinability.

Acknowledgments. This work is supported by the National Key Basic Research and Development Program (973 Program) of China (Grant No. 2011CB706702), Natural Science Foundation of China (Grant Nos. 51305161 and 51135006), Jilin province science and technology development plan item (Grant No. 20130101042JC) and 863 Program (Grant Nos. 2012AA041304 and 2012AA040406).

1. W. A. Glaeser, Materials for Tribology, Elsevier Science, New York (1992).

2. K. Friedrich, Advances in Composite Tribology, Elsevier Science, New York (1993).

3. M. Rahman, S. Ramakrishna, and H. C. Thoo, "Machinability study of carbon/PEEK composites," Mach. Sci. Technol., 3, 49-59 (1999). 
4. S. Jahanmir, M. Ramulu, and P. Koshy, Machining of Ceramics and Composites, Marcel Dekker, Inc., New York (1998).

5. J. D. Paulo, P. Reis, and V. Lapa, "Machinability study on polyetheretherketone (PEEK) unreinforced and reinforced (GF30) for applications in structural components," Compos. Struct., 62, No. 1, 67-73 (2003).

6. D. D. L. Chung, Composite Materials, Springer (2010).

7. Z. Rasheva, G. Zhang, and T. Burkhart, "A correlation between the tribological and mechanical properties of short carbon fibers reinforced PEEK materials with different fiber orientations," Tribol. Int., 43, No. 8, 1430-1437 (2010).

8. D. J. Blundell and B. N. Osborn, "Crystalline morphology of the matrix of PEEKcarbon fiber aromatic polymer composites. Part II. Crystallization behavior," SAMPE Quart., 17, No. 1, 1-6 (1985).

9. P. Cebe and S. D. Hong, "Crystallization behaviour of poly (ether-ether-ketone)," Polymer, 27, No. 8, 1183-1192 (1986).

10. A. Jonas and R. Legras, "Relation between PEEK semicrystalline morphology and its subglass relaxations and glass transition," Macromolecules, 26, No. 4, 813-824 (1993).

11. M. F. Talbott, G. S. Springer, and L. A. Berglund, "The effects of crystallinity on the mechanical properties of PEEK polymer and graphite fiber reinforced PEEK," $J$. Compos. Mater., 21, No. 11, 1056-1081 (1987).

12. P. J. Rae, E. N. Brown, and E. B. Orler, "The mechanical properties of poly (ether-ether-ketone) (PEEK) with emphasis on the large compressive strain response," Polymer, 48, No. 2, 598-615 (2007).

13. D. P. Jones, D. C. Leach, and D. R. Moore, "Mechanical properties of poly(etherether-ketone) for engineering applications," Polymer, 26, No. 9, 1385-1393 (1985).

14. P. S. Allan and M. J. Bevis, Process for Molding Directionally-Orientatable Material Using Shear Force, Patent US 4925161 A (1990).

15. S. Sato, W. Gao, and S. Kiyono, "Design of a precision rotary-linear dual-axis positioning system with a surface encoder," Proc. SPIE 6052, Optomechatronic Systems Control, 60520J (December 06, 2005), DOI: 10.1117/12.647931.

16. D. P. Yu, Y. S. Wong, and G. S. Hong, "Ultraprecision machining of micro-structured functional surfaces on brittle materials," J. Micromech. Microeng., 21, No. 9, 095011 (2011).

17. Z. W. Zhu, X. Q. Zhou, and Q. Liu, "Fabrication of micro-structured surfaces on bulk metallic glasses based on fast tool servo assisted diamond turning," Sci. Adv. Mater., 4, No. 9, 906-911 (2012). 